\title{
Antithrombotic therapy in acute coronary syndrome: undertreatment of elderly?
}

\author{
Iacopo Muraca, Francesca Ciatti, Angela Migliorini, Niccolò Marchionni, Renato Valenti \\ Cardiovascular Department, Careggi University Hospital, Florence, Italy
}

\begin{abstract}
The appropriate use of dual antiplatelet therapy (DAPT) in elderly patients with acute coronary syndrome (ACS) is largely debated. Due to the "demographic transition" in western countries, the elderly represent a growing percentage of patients admitted to hospitals for ACS, and among this high risk setting population most of early and late adverse outcomes occur. The presence of several concomitant "comorbidities" complicates the management strategy of both medical or invasive treatment and the clinical decision-making process is challenging. Moreover, elderly
\end{abstract}

\footnotetext{
Correspondence: Renato Valenti, Interventional Cardiology Unit, Cardiovascular Department, Careggi University Hospital, Largo Brambilla 3, 50141 Florence, Italy.

Tel. +39.055.7949780.

E-mail: renato.valenti2@tin.it
}

Keywords: Elderly; antithrombotic therapy; frailty; multimorbidity; acute coronary syndrome.

Author's contribution: RV, study concept, manuscript drafting; IM, FC, data revision and interpretation, manuscript drafting; AM, NM, substantial intellectual contribution, manuscript critical revision. All the authors have read and approved the final version of the manuscript and agreed to be accountable for all aspects of the work.

Ethics approval and consent to participate: No ethical committee approval was required for this study, because this article does not contain any studies with human participants or animals.

Conflict of interest: none declared.

Acknowledgments: The authors are indebted to Fabio Torrini and Paola Baldini (A.R. Card Onlus Foundation, Florence, Italy) for their precious help and secretarial assistance.

Received for publication: 1 March 2020.

Accepted for publication: 22 April 2020.

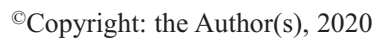

Licensee PAGEPress, Italy

Monaldi Archives for Chest Disease 2020; 90:1261

doi: 10.4081/monaldi.2020.1261

This article is distributed under the terms of the Creative Commons Attribution Noncommercial License (by-nc 4.0) which permits any noncommercial use, distribution, and reproduction in any medium, provided the original author(s) and source are credited. people are constantly underrepresented in clinical trials and studies. As a result, there is no specific evidence about the optimal antithrombotic therapy in elderly and no specific recommendations are mentioned in the current ACS guidelines. Currently, the best practice for old people is still rudimentary and principally extrapolated from general cardiovascular guidelines.

The management of elderly patients should be based on the evaluation of ischemic and hemorrhagic risk, life expectancy, comorbidities and parameters that are not included in the scores recommended by the current guidelines such as frailty and disability. In the era of "precision medicine", the evaluation of bleeding and ischemic risk in elderly patients must be a dynamic process because of the risk changing over time. A "tailored therapy by individualized medicine" is the key of management strategy.

\section{Prevalence and incidence of coronary artery disease in elderly}

The average life expectancy is increased by nearly 30 years during the last century [1]. Data from the Centers for Disease Control and Prevention shows that the main contribution to the increase in life expectancy is due to the improvement of prevention and treatment of cardiovascular disease (CVD). The decline in CVD was due mainly to secondary prevention with primary prevention accounting for only one quarter [2].

Prevalence and incidence of coronary artery disease (CAD) increase with advancing age and currently "elderly" people is defined by a cut-off of $\geq 75$ years $[3,4]$. Elderly people are generally affected by "multimorbidity". This term describes what is commonly observed in clinical practice when clinicians cannot identify any dominant disease and must deal with several coexisting illnesses at the same time. Moreover, the presence of multiple clinical diseases in elderly increase the likelihood of adverse clinical outcomes [5]. Furthermore, older age often overlaps with a condition of frailty and disability [6]. Briefly, the current definition of "frailty" is a state of progressive deterioration related to ageing that generates a greater vulnerability to stressors and this correlates with higher probability of adverse events in terms of disability and mortality. The prevalence of frailty in elderly with CVD rises up to $60 \%$ and is about 3 times more prevalent compared to the other older adults in the community [7]. As expected, acute coronary syndrome (ACS) in elderly is associated to a poor prognosis when compared with younger population; the management and the decision-making process of antithrombotic therapies in elderly are challenging due to an increased risk of both bleeding and ischemic events.

Currently, guidelines include scores supporting clinicians for 
assessing intensity and duration of antithrombotic therapy, but these tools do not consider important factors such as frailty and disability. However, recently published data from a small cohort of patients, showed that frailty or physical performance scales were able to discriminate the occurrence of bleeding events, but when integrated with bleeding scores did not result in any clinically meaningful improvement in the prediction of bleedings [8]. Furthermore, bleeding scores mentioned probably overweight agerelated patient bleeding risk [9].

\section{Undertreatment of elderly patients in acute coronary syndrome}

The appropriate use of dual antiplatelet therapy (DAPT) in elderly patients with ACS is largely debated. In patients with ACS, risk factors associated with bleeding and ischemic events tend to overlap and the correct balance between ischemic and hemorrhagic risk is more difficult to assess in elderly than in young people, especially when considering the use of more potent antithrombotic drug therapy.

Despite recent guideline recommendations, evidence-based therapies for ACS are less prescribed and focusing on antithrombotic management, antiplatelet therapy is currently underutilized in elderly patients [10]. Real world and registries data report that older patients are systematically undertreated due to their increasing comorbidities $[11,12]$. In contrast to younger patients in whom CVD typically presents as a dominant medical condition, in elderly is part of multiple chronic conditions. Moreover, interactions among conditions and treatments in patients with comorbidity, dealing of a treatment efficacy and its adverse effects may be further challenged by shortened life expectancy and by the fatality of ACS with advancing age. This concern is due to an improperly perceived poor risk/benefit ratio, raising concerns about "ageism" [13].

Several trials have reported that in elderly, percutaneous coronary revascularization for ACS is affected by higher rate of complication and adverse outcome; conversely, others studies, registries and observational studies proved that an "aggressive treatment" is more effective, providing a higher "net clinical benefit" with increasing age [14].

The clinical decision-making of any therapeutic strategy in elderly patients is further complicated by the context of physiological age-related organ changes that affect the pharmacokinetic and pharmacodynamic process. There is a slightly decreased absorption, a different drug distribution according to different body's composition, a less effective hepatic metabolism and an impaired renal elimination.

Recent evidences showed that advanced age is associated to high on-treatment platelet reactivity (HPR) in patients treated with clopidogrel; HPR is present up to $40 \%$ of elderly patients [15]. Moreover, studies have reported that HPR in patients receiving clopidogrel is associated with a worse prognosis at long-term follow-up [16]. Several explanations have been hypothesized for the reduced efficacy of clopidogrel in elderly patients: a poor conversion of its metabolites due to a decrease in activity of the liver enzyme CYP450 in older adults and/or a deficit in platelet turnover. Considering the pathophysiology of ACS, which involves platelet aggregation, the presence/persistence of HPR is associated with a worse prognosis [17]. The results of the CURE trial showed that the benefit of treatment with ASA and clopidogrel in terms of reduction of the combined primary endpoint (death from cardiovascular causes, myocardial infarction, stroke) is also maintained in elderly patients ( $>65$ years) [18]. However, as suggested by the SENIOR-PLATELET study, age is a strong predictor of HPR during clopidogrel therapy, resulting in a reduced anti platelet activity of the drug [19].

These reasons support the use of new and more potent antiplatelet agents in elderly patients, always balancing the increased hemorrhagic risk. Data from clinical trials and some meta-analysis conducted on new antiplatelet agents have also shown an advantage in the sub-group of older patients with regard to ischemic events and mortality that tend to increase with increasing age. In particular, the PLATO study showed a reduction of the primary efficacy end point (death and ischemic events) of $18 \%$ in patients over 75 years compared to $10 \%$ of patients under 75 years treated with ticagrelor $[20,21]$. In contrast, the study TRITONTIMI 58 with prasugrel showed a decrease in the net clinical benefit in the elderly patient linked to a greater number of bleedings, suggesting a dose reduction in patients over 75 years. A further study, showed no superiority of reduced-dose of prasugrel compared to the standard-dose of clopidogrel in patients undergoing early percutaneous revascularization [22]. Similarly, in the TRILOGY-ACS trial, no differences in ischemic or bleeding outcomes were found even in medically managed elderly ACS patients $[23,24]$. These data support the potential benefit of ticagrelor as a drug of choice in this subgroup of patients requiring DAPT, if not contraindicated.

However, in the real word, elderly patients are often undertreated, especially regarding the use of newer P2Y12 antiplatelet drugs, due to an improperly perceived closer correlation between advanced age and bleeding rather than ischemic risk. In contrast to this wrong perception, recent data shows that patients with two or more hemorrhagic-ischemic risk factors have increasing rates of ischemic events, and less incidence of bleeding events. In particular, excluding history of prior bleeding, the increasing number of comorbidities were associated with a more significant increase of ischemic events as compared to bleedings [25]. This highlight the importance for assessing patient bleeding history, which could have implications in clinical decision-making regarding intensity and duration of secondary antithrombotic preventive treatment. Current guidelines suggest a more conservative approach in patients with a PRECISE-DAPT score value $\geq 25$ [26], but the vast majority of elderly patients have PRECISE-DAPT values above the recommended cut-off point for bleeding risk. Indeed, elderly patients have higher prevalence of diabetes mellitus, previous revascularization, more extensive coronary artery disease or left main disease, and therefore are usually at higher risk for recurrent ischemic events. Using different cut-off points in older patients could probably be a more rational approach for predicting bleeding risk in these complex patients [27].

Regarding duration of DAPT, patient's age itself should not be an indication to a shorter DAPT regimen [28]. Otherwise, a history of previous bleeding is a warning for long-term DAPT and a shorter regimen may be recommended in frail patients even if there is no specific evidence about the optimal antiplatelet therapy and no specific recommendation is mentioned in the current ACS guidelines. DAPT is a systemic treatment strategy able to convey in a global patient protection. The trade-off between the benefit and risk of DAPT may differ from that observed in selected patients included in randomized trials, since elderly people are constantly underrepresented in clinical trials and studies.

Finally, it is true that older patients have multi-organ changes, increased risk of both bleeding and ischemic events, frequent comorbidities/comedication, and reduced adherence to prescriptions, but for these reasons we must not commit "ageism". 


\section{Conclusions}

Elderly represent a subgroup of high-risk ACS patients due to their advanced age and other comorbidities, where clinical decision-making process is particularly challenging. Despite the availability of proven scientific evidence, elderly patients may not receive optimal treatment for ACS. There is a need for the implementation of strategies aimed to incorporate evidence into clinical practice that expand the use of proven, effective medical therapy or invasive strategy focusing on elderly patients, with the aim of reducing the burden of ACS. The decision-making process should be based on the evaluation of ischemic and hemorrhagic risk, life expectancy, comorbidities, parameters that are not included in the scores recommended by the current guidelines such as frailty and disability, in addition to quality of life, preference of the patient as well as the estimated risk and benefit of coronary revascularization.

In elderly patient the evaluation of bleeding risk must be a dynamic process because the risk can vary over time; a "tailored therapy by individualized precision medicine" is the key of the management strategy. Therefore, it is important not only to categorize patients based on the risk of bleeding, but also to follow them through careful follow-up.

We must integrate data from studies, trials, guidelines, recommendations and clinical judgment, making an ongoing assessment to navigate the best strategy for each patient. Balancing the expected benefits and harms is crucial to decide the right management.

\section{References}

1. Lenfant C. Shattuck lecture: Clinical research to clinical practice - Lost in translation? N Engl J Med 2003;349:868-74.

2. Cutler DM, Rosen AB, Vijan S. The value of medical spending in the United States, 1960-2000. N Engl J Med 2006;355:920-7.

3. D'Agostino RB, Vasan RS, Pencina MJ, et al. General cardiovascular risk profile for use in primary care: The Framingham heart study. Circulation 2008;117:743-53.

4. Andreotti F, Rocca B, Husted S, et al. Antithrombotic therapy in the elderly: Expert position paper of the European society of cardiology working group on thrombosis. Eur Heart J 2015;36:3238-49.

5. Forman DE, Maurer MS, Boyd C, et al. Multimorbidity in older adults with cardiovascular disease. J Am Coll Cardiol 2018;71:2149-61.

6. Ungar A, Marchionni N. Cardiac management in the frail elderly patient and the oldest old. Springer; 2017; 177 p.

7. Afilalo J, Karunananthan S, Eisenberg MJ, et al. Role of frailty in patients with cardiovascular disease. Am J Cardiol 2009;103:1616-21.

8. Pavasini R, Maietti E, Tonet E, et al. Bleeding risk scores and scales of frailty for the prediction of haemorrhagic events in older adults with acute coronary syndrome: Insights from the FRASER study. Cardiovasc Drugs Ther 2019;33:523-32.

9. Ariza-Solé A, Guerrero C, Formiga F, et al. Global geriatric assessment and in-hospital bleeding risk in elderly patients with acute coronary syndromes: Insights from the LONGEVOSCA Registry. Thromb Haemost 2018;118:581-90.

10. Alexander KP, Newby LK, Cannon CP, et al. Acute coronary care in the elderly, part I. Non-ST-segment-elevation acute coronary syndromes: A scientific statement for healthcare professionals from the American heart association council on clinical cardiology. Circulation 2007;115:2549-69.

11. Radovanovic D, Seifert B, Urban P, et al. Validity of Charlson
Comorbidity Index in patients hospitalised with acute coronary syndrome. Insights from the nationwide AMIS Plus registry 2002-2012. Heart 2014; 100:288-94.

12. Cirillo P, Di Serafino L, Taglialatela V, et al. Optimal medical therapy on top of dual-antiplatelet therapy: 1-year clinical outcome in patients with acute coronary syndrome: The START Antiplatelet Registry. Angiology 2020;71:235-41.

13. Strandberg T, Pietikäinen S, Maggi S, et al. Against age discrimination. Lancet 2015;386:337-8.

14. Di Bari M, Balzi D, Fracchia S, et al. Decreased usage and increased effectiveness of percutaneous coronary intervention in complex older patients with acute coronary syndromes. Heart 2014;100:1537-42.

15. Verdoia M, Pergolini P, Rolla R, et al. Advanced age and highresidual platelet reactivity in patients receiving dual antiplatelet therapy with clopidogrel or ticagrelor. J Thromb Haemost 2016;14:57-64.

16. Parodi G, Marcucci R, Valenti R, et al. High residual platelet reactivity after clopidogrel loading and long-term cardiovascular events among patients with acute coronary syndromes undergoing PCI. J Am Med Assoc 2011;306:1215-23.

17. De Rosa R, Palmerini T, De Servi S, et al. High on-treatment platelet reactivity and outcome in elderly with non ST-segment elevation acute coronary syndrome - Insight from the GEPRESS study. Int J Cardiol 2018;259:20-5.

18. Yusuf S, Zhao F, Mehta SR, et al. Effects of clopidogrel in addition to aspirin in patients with acute coronary syndromes without ST-segment elevation. N Engl J Med 2001;345:494-502.

19. Silvain J, Cayla G, Hulot JS, et al. High on-thienopyridine platelet reactivity in elderly coronary patients: The SENIORPLATELET study. Eur Heart J 2012;33:1241-50.

20. Tarantini G, Ueshima D, D'Amico G, et al. Efficacy and safety of potent platelet $\mathrm{P} 2 \mathrm{Y} 12$ receptor inhibitors in elderly versus nonelderly patients with acute coronary syndrome: A systematic review and meta-analysis. Am Heart J 2018;195:78-85.

21. Madhavan MV, Gersh BJ, Alexander KP, et al. Coronary artery disease in patients $\geq 80$ years of age. J Am Coll Cardiol 2018;71:2015-40.

22. Savonitto S, Ferri LA, Piatti L, et al. Comparison of reduceddose prasugrel and standard-dose clopidogrel in elderly patients with acute coronary syndromes undergoing early percutaneous revascularization. Circulation 2018;137:2435-45.

23. Roe MT, Armstrong PW, Fox KAA, et al. Prasugrel versus clopidogrel for acute coronary syndromes without revascularization. N Engl J Med 2012;367:1297-309.

24. Roe MT, Goodman SG, Ohman EM, et al. Elderly patients with acute coronary syndromes managed without revascularization: Insights into the safety of long-term dual antiplatelet therapy with reduced-dose prasugrel versus standard-dose clopidogrel. Circulation 2013;128:823-33.

25. Lindholm D, Sarno G, Erlinge D, et al. Combined association of key risk factors on ischemic outcomes and bleeding in patients with myocardial infarction. Heart 2019;105:1175-81.

26. Valgimigli M, Bueno H, Byrne RA, et al. 2017 ESC focused update on dual antiplatelet therapy in coronary artery disease developed in collaboration with EACTS. Eur Heart J 2018;39:213-60.

27. Guerrero C, Ariza-Solé A, Formiga F, et al. Applicability of the PRECISE-DAPT score in elderly patients with myocardial infarction. J Geriatr Cardiol 2018;15):713-7.

28. Gulizia MM, Colivicchi F, Abrignani MG, et al. Consensus document ANMCO/ANCE/ARCA/GICR-IACPR/GISE/SICOA: Long-term antiplatelet therapy in patients with coronary artery disease. Eur Hear J 2018;20:F1-F74. 